\title{
The role of inflammation markers in predicting the prognosis of Bell's palsy
}

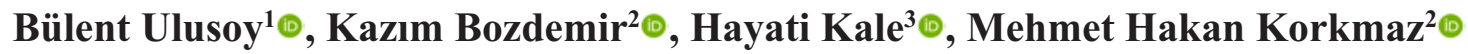 \\ ${ }^{1}$ Department of Otorhinolaryngology-Head and Neck Surgery, Selçuk University School of Medicine, Konya, Turkey \\ ${ }^{2}$ Department of Otorhinolaryngology-Head and Neck Surgery, Ylldırım Beyazlt University School of Medicine, Ankara, Turkey \\ ${ }^{3}$ Department of Otorhinolaryngology-Head and Neck Surgery, Dr. Sami Ulus Training and Research Hospital, Ankara, Turkey
}

DOI: $10.18621 /$ eurj.404778

\begin{abstract}
Objectives: This study aimed to investigate the relationship between the neutrophil-to-lymphocyte ratio (NLR) and the platelet-to-lymphocyte ratio (PLR) and Bell's palsy in addition to the usability of these markers to predict prognosis.

Methods: In this prospective study, the patient group included 24 patients that were diagnosed with Bell's palsy and 29 healthy volunteers that had similar characteristics to them regarding age and gender with patient group as the control group. The temporal gadolinium-enhanced magnetic resonance imaging (TGd-MRI) was performed to all patients. The complete blood count was also obtained from both the patient and the control groups, and these two groups were compared for NLR and PLR, mean platelet volume (MPV), platelet distribution width (PDW), and platelet count. The relationship between these markers and Bell's paralysis prognosis was also investigated.

Results: The patient and control groups were similar in age and gender $(p>0.05)$. NLR was significantly higher in the patient group compared with the control group $(p=0.016)$. There was no correlation between the NLR value and the prognosis of Bell's Palsy. The PDW and white blood cell count (WBC) were significantly higher in the patient group than the control group ( $p=0.023$ and $p=0.012$, respectively). A comparison of the recovered and unrecovered patients revealed that the PDW value was significantly higher and the platelet count was lower in the recovered patients than the unrecovered patients ( $p=0.009$ and $p=0.003$ respectively). The MPV value was higher in the recovered patients, though not statistically significant $(p=0.063)$. Based on the cut-off values determined in the receiver operator characteristic (ROC) curve analysis, a comparison of the recovered and unrecovered patients reveals that the likelihood of not healing was significantly higher in those that had normal PDW values $(p=0.036)$.
\end{abstract}

Conclusion: These results might support the theory of inflammation in patients with Bell's palsy.

Keywords: Bell palsy, inflammation, mean platelet volume, prognosis, platelet activation, ROC curve, neutrophil

Received: March 12, 2018; Accepted: August 15, 2018; Published Online: September 14, 2018

B ell's palsy is defined as an acute peripheral facial palsy, usually affecting one half of the face [1, 2]. The facial nerve provides the sense of a specific part of the auriculus, the sense of taste in two-thirds of the anterior tongue, and the autonomous innervation of the lacrimal and mandibular glands in addition to

Address for correspondence: Bülent Ulusoy, MD., Assistant Professor, Selçuk University School of Medicine, Department of OtorhinolaryngologyHead and Neck Surgery, Konya, Turkey

E-mail: ulusoybulent@hotmail.com,Tel:+90 332 2412181-43920,Fax:+90332 2412184 
the motor innervation of the facial muscles; thus, there are certain symptoms and findings (e.g., sudden-onset visual impairment on one side of the faces, dry eye, and incomplete eyes and mouth closure) emerging in relation to these functions [1]. Bell's palsy has an annual incidence of 15-30/100,000, which corresponds to $60-75 \%$ of all unilateral facial palsies [2]. Some patients reported being exposed to the air condition or an open window before the paralysis [1]. Although the etiology of facial paralysis is still unclear, viral infection, vascular ischemia, or autoimmunity are thought to be the causes of this disease $[1,2]$.

The neutrophil-to-lymphocyte ratio (NLR) and the platelet-to-lymphocyte ratio (PLR) have recently been used frequently as a new marker of inflammation [3-5]. This study aimed to investigate the relationship between NLR and PLR values and Bell's palsy, and the usability of these markers to predict the prognosis.

\section{METHODS}

This study was conducted with 24 patients that were diagnosed with Bell's palsy between the years 2015 and 2016, and 29 healthy volunteers (the control group) with similar age and gender characteristics. The exclusion criteria included the following: patients with acute infection, diabetes mellitus, systemic hypertension, hyperlipidemia, acute or chronic renal failure, chronic liver disease, chronic obstructive pulmonary disease, coronary artery disease, connective tissue disease, inflammatory bowel disease, allergic rhinitis, smoking history, chronic otitis media and otosclerosis, and other otic diseases such as acoustic trauma, patients who are performed otological surgery before, and tumors or cochlear pathology detected on the temporal gadoliniumenhanced magnetic resonance imaging (TGd-MRI).

The stage of Bell's palsy was determined according to the House-Brackmann staging system [2] at the time of diagnosis and 1 month after the treatment. A complete ear-nose-throat and head and neck examinations were performed for all patients. Some tests, such as the complete blood count (CBC), pure tone audiometry, stapes reflex, routine blood biochemistry, and viral serological markers, were obtained from the patients diagnosed with unilateral peripheral facial paralysis before the treatment.
Additionally, TGd-MRI was obtained from all patients at the onset of the treatment. In the control group was performed only CBC.

A systemic steroid (methylprednisolone) treatment at a dose of $1 \mathrm{mg} / \mathrm{kg}$ was started routinely for patients diagnosed with Bell's palsy, and the dose was reduced and terminated in 10 days. At the end of the treatment, the House-Brackmann grade 1-2 was regarded as a satisfactory recovery and grade 3-6 was regarded as unsatisfactory recovery [3]. Patients who did not change according to the pre-treatment stage were defined as unrecovered.

The patient and control groups were compared for NLR, PLR, mean platelet volume (MPV), and platelet distribution width (PDW). These markers were also evaluated for their usability in predicting the prognosis.

\section{Hematological Analysis}

NLR was calculated as a simple ratio between the absolute neutrophil and the absolute lymphocyte counts in the CBC. PLR was also calculated as a simple ratio between the absolute platelet count and the absolute lymphocyte count. Moreover, the MPV and PDW values were determined from the CBC. The NLR, PLR, MPV, and PDW values were calculated in the control group in a similar manner. An automated blood cell counter (Sysmex XE-2100; Kobe, Japan) was used for the analyses.

\section{Approval of the Research Ethics Committee}

The study was carried out with the permission (26379996/177) of the Clinical Research Ethics Committee.

\section{Statistical Analysis}

SPSS for Windows 11.5 package program (Chicago, Inc, USA) for statistical analysis was used. In the analyses, independent samples t test was used for the comparison of the variables in two groups as well as the variables with normal distribution. MannWhitney U Test was used for the variables that did not have a normal distribution.

The receiver operator characteristics (ROC) curve analysis was applied to determine the most appropriate cut-off values for the statistically significant variables among the groups. The chi-square and Fisher's exact tests were used to compare the cut-off values obtained 
Table 1. Comparison between patient and control groups according to age and sex

\begin{tabular}{lccc}
\hline & Control $(\mathbf{n}=\mathbf{2 9})$ & Patient $(\mathbf{n}=\mathbf{2 4})$ & p value \\
\hline $\begin{array}{l}\text { Sex } \\
\text { Male/Female }\end{array}$ & $13 / 16$ & $13 / 11$ & 0.498 \\
$\begin{array}{l}\text { Age (years) } \\
\text { (Mean } \pm \text { SD) }\end{array}$ & $44.34 \pm 9.97$ & $45.12 \pm 12.34$ & 0.800 \\
\hline SD $=$ standard deviation & &
\end{tabular}

$\mathrm{SD}=$ standard deviation

as a result of these analyses. The statistical significance threshold was accepted as 0.05 .

\section{RESULTS}

The mean age was $45.12 \pm 12.34$ years in the patient group and $44.34 \pm 9.97$ years in the control group with no statistically significant difference between these two groups $(p>0.05)$. The female/male ratio was $13 / 16$ in the control group and $13 / 11$ in the patient group, and when the groups compared according to gender were similar. $(p>0.05)$ (Table 1$)$. Five patients who did not participate in the control examination after the treatment were excluded from the study. Satisfactory recovery was detected in 16 patients. Since the stage of the disease did not change before and after the treatment in three patients, they were classified under the unrecovered group.

The mean NLR was $2.86 \pm 2.01$ in the patient group and $1.68 \pm 0.34$ in the control group, and NLR was found to be significantly higher in the patient group compared with the control group ( $p=0.016$ ). The PDW value was $13.46 \pm 2.40 \mathrm{fl}$ in the patient group and $12.31 \pm 1.02 \mathrm{fl}$ in the control group. The white blood cell $(\mathrm{WBC})$ count was $9.13 \pm 3.33\left(\times 10^{9} / 1\right)$ in the patient group and $7.40 \pm 1.22\left(\times 10^{9} / 1\right)$ in the control group. The PDW and WBC values were significantly higher in the patient group compared with the control group ( $p=0.023$ and $p=0.012$, respectively). The PLR value was $121.90 \pm 52.87$ in the patient group and $99.98 \pm 16.93$ in the control

Table 2. Comparison of the patient and control groups in terms of variables

\begin{tabular}{lccc}
\hline & Control $(\mathbf{n}=\mathbf{2 9})$ & Patient $(\mathbf{n}=\mathbf{2 4})$ & p value \\
\hline PLR & $99.98 \pm 16.93$ & $121.90 \pm 52.87$ & 0.133 \\
NLR & $1.68 \pm 0.34$ & $2.86 \pm 2.01$ & $\mathbf{0 . 0 1 6}$ \\
MPV (fl) & $10.46 \pm 0.54$ & $10.23 \pm 1.25$ & 0.378 \\
Platelet count $(\times \mathbf{1 0} / \mathbf{l})$ & $247.59 \pm 43.20$ & $245.58 \pm 62.44$ & 0.891 \\
PDW (fl) & $12.31 \pm 1.02$ & $13.46 \pm 2.40$ & $\mathbf{0 . 0 2 3}$ \\
WBC $(\times \mathbf{1 0} / \mathbf{l})$ & $7.40 \pm 1.22$ & $9.13 \pm 3.33$ & $\mathbf{0 . 0 1 2}$ \\
\hline
\end{tabular}

Data are shown as mean \pm standard deviation. MPV $=$ mean platelet volume, NLR $=$ neutrophil-tolymphocyte ratio, $\mathrm{PDW}=$ platelet distribution width, $\mathrm{PLR}=$ platelet-to-lymphocyte ratio, $\mathrm{WBC}=$ white blood cell

Table 3. Comparison of the recovery results in the patient group

\begin{tabular}{lccc}
\hline & Present $(\mathbf{n}=\mathbf{1 6})$ & Absent $(\mathbf{n}=\mathbf{3})$ & p value \\
\hline PLR & $132.16 \pm 58.83$ & $92.96 \pm 30.89$ & 0.211 \\
NLR & $2.89 \pm 2.28$ & $1.90 \pm 0.68$ & 0.793 \\
MPV (fl) & $10.30 \pm 1.11$ & $8.90 \pm 1.15$ & $\mathbf{0 . 0 6 3}$ \\
Platelet counts $(\times \mathbf{1 0} / \mathbf{l})$ & $240.63 \pm 55.01$ & $289.00 \pm 2.00$ & $\mathbf{0 . 0 0 3}$ \\
PDW $(\mathbf{f l}){ }^{9}$ & $13.91 \pm 1.80$ & $10.63 \pm 1.69$ & $\mathbf{0 . 0 0 9}$ \\
WBC $(\times \mathbf{1 0} / \mathbf{l})$ & $8.56 \pm 3.62$ & $10.12 \pm 1.79$ & 0.481 \\
\hline
\end{tabular}

Data are shown as mean \pm standard deviation. MPV $=$ mean platelet volume, NLR = neutrophil-to-lymphocyte ratio, $\mathrm{PDW}=$ platelet distribution width, $\mathrm{PLR}=$ platelet-to-lymphocyte ratio, $\mathrm{WBC}=$ white blood cell 
Table 4. ROC analysis results for NLR, PDW, and WBC.

\begin{tabular}{lccc}
\hline & NLR & PDW & WBC \\
\hline AUC & 0.6936 & 0.670 & 0.671 \\
SE & 0.082 & 0.081 & 0.088 \\
$\boldsymbol{p}$ value & $\mathbf{0 . 0 1 6}$ & $\mathbf{0 . 0 3 4}$ & $\mathbf{0 . 0 3 3}$ \\
$\begin{array}{l}\text { Cut-off } \\
\text { point }\end{array}$ & 1.80 & 12.60 & 8.28 \\
\hline
\end{tabular}

$\mathrm{AUC}=$ area under the $\mathrm{ROC}$ curve, $\mathrm{ROC}=$ receiver operator characteristics

group, but the difference was not statistically significant $(p>0.05)$. Similarly, no significant difference was found between the patient and control groups in terms of MPV and platelet count $(p>0.05)$ (Table 2).

The patient group was categorized into two subgroups: recovered and unrecovered. The PDW value was $13.91 \pm 1.80 \mathrm{fl}$ in the recovered group and $10.63 \pm$ $1.69 \mathrm{fl}$ in the unrecovered group $(p=0.009)$. On the contrary, the platelet count was $240.63 \pm 55.1\left(\times 10^{9} / 1\right)$ in the recovered group and $289.00 \pm 2.00\left(\times 10^{9} / 1\right)$ in the unrecovered group $(p=0.003)$. Interestingly, the study determined that the PDW value was significantly higher and the platelet count was lower ( $p=0.003$ and $p=0.009$, respectively) in the recovered patients when the patient group was divided into two groups as recovered and unrecovered individuals. Additionally, the MPV value was higher in the recovered group but this result was not statistically significant ( $p=0.063$ ) (Table 3).

When the patient and control groups were taken into consideration, statistically significant cut-off values were determined for NLR, PDW, and WBC in the ROC analysis that was performed to determine the most appropriate cut-off values. The results were pathologically determined as NLR $>1.8, \mathrm{PDW}>12.6$ fl, and WBC $>8.28\left(\times 10^{9} / 1\right)($ Figure 1 , Table 4$)$. The pathological rates of NLR, PDW, and WBC were higher in the patient group (Table 5). No significant difference was obtained in the NLR and WBC values between recovered and unrecovered subgroups in the patient group; however, the possibility of not recovering was found to be significantly higher in the patients having normal PDW levels (Table 6).

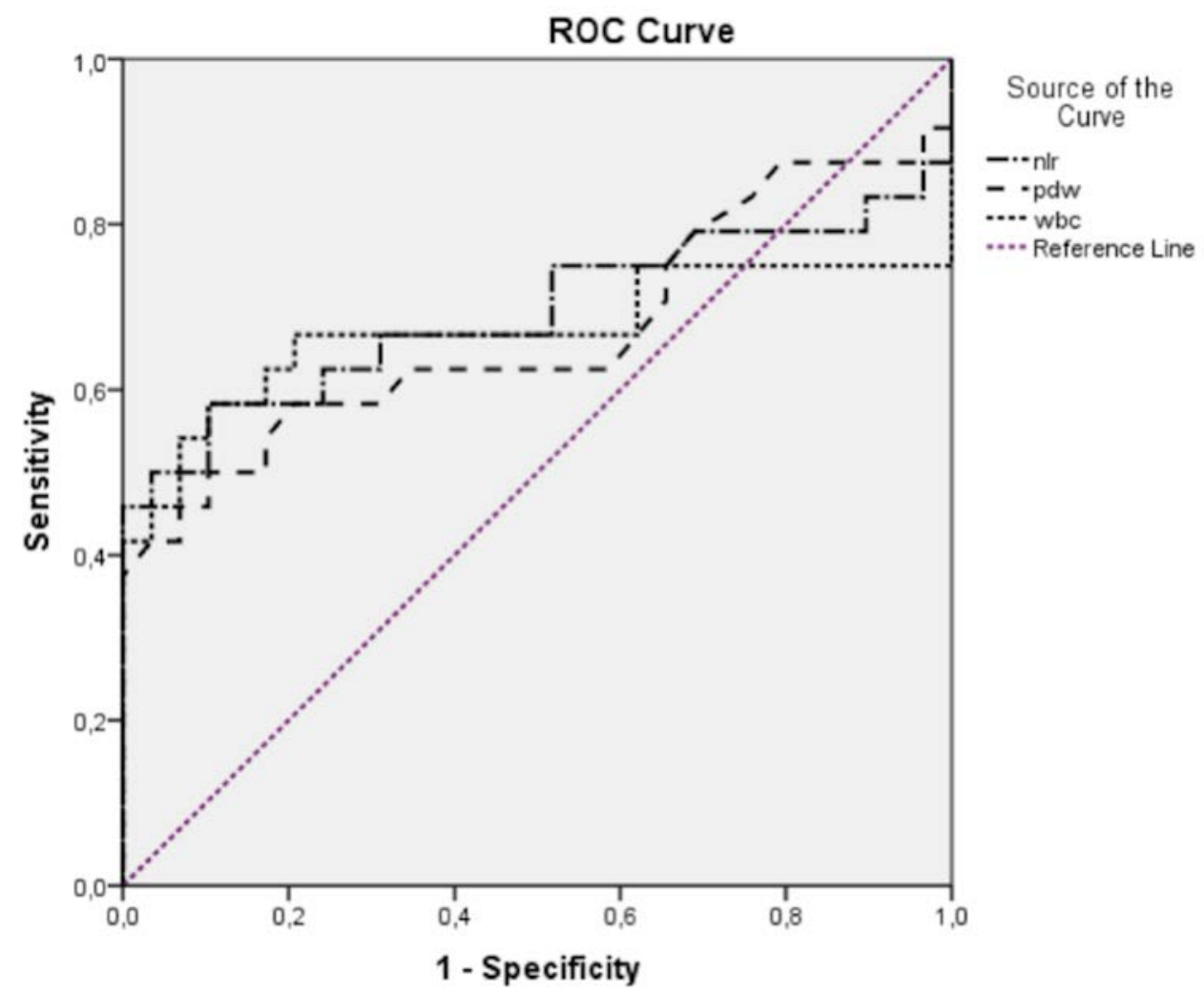

Figure 1. ROC analysis results for NLR, PDW, and WBC. 
Table 5. Control and patient group comparison results according to the cut-off values for NLR, PDW, and WBC

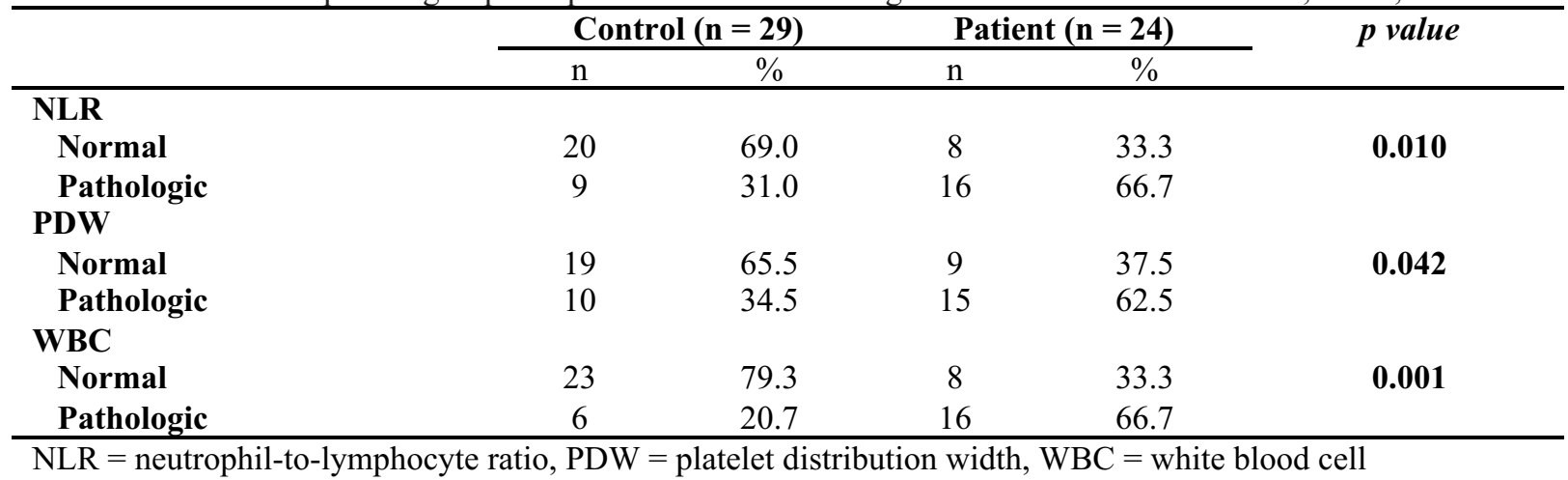

$\mathrm{NLR}=$ neutrophil-to-lymphocyte ratio, $\mathrm{PDW}=$ platelet distribution width, $\mathrm{WBC}=$ white blood cell

Table 6. Comparison of cut-off values for NLR, PDW, and WBC according to the recovery status in the patient group

\begin{tabular}{|c|c|c|c|c|c|}
\hline & \multicolumn{2}{|c|}{ Present $(n=16)$} & \multicolumn{2}{|c|}{ Absent $(n=3)$} & \multirow[t]{2}{*}{ p value } \\
\hline & $\mathrm{n}$ & $\%$ & $\mathrm{n}$ & $\%$ & \\
\hline \multicolumn{6}{|l|}{ NLR } \\
\hline Normal & 6 & 85.7 & 1 & 14.3 & \multirow[t]{2}{*}{0.704} \\
\hline Pathologic & 10 & 83.3 & 2 & 16.7 & \\
\hline \multicolumn{6}{|l|}{ PDW } \\
\hline Normal & 4 & 57.1 & 3 & 42.9 & \multirow[t]{2}{*}{0.036} \\
\hline Pathologic & 12 & 100.0 & 0 & 0.0 & \\
\hline \multicolumn{6}{|l|}{ WBC } \\
\hline Normal & 7 & 100.0 & 0 & 0.0 & \multirow[t]{2}{*}{0.227} \\
\hline Pathologic & 9 & 75.0 & 3 & 25.0 & \\
\hline
\end{tabular}

$\mathrm{NLR}=$ neutrophil-to-lymphocyte ratio, $\mathrm{PDW}=$ platelet distribution width, $\mathrm{WBC}=$ white blood cell

\section{DISCUSSION}

Bell's palsy is defined as a peripheral facial paralysis with a sudden onset, involving one half of the face. Its incidence has peaked in the last two to four decades. Although this occurs equally in males and females, a slight female predominance was seen in the previous studies $[1,2]$. Although the etiology of facial paralysis has not been fully understood yet, infection, microcirculation, circulatory insufficiency, genetic, and immunological and inflammatory factors are thought to play a role in its occurrence $[1,2,6]$. Past studies reported that autoimmune mechanisms play an important role in the pathogenesis of Bell's palsy, and interleukin-1 (IL-1), IL-6, IL-8 and tumor necrosis factor- $\alpha$ (TNF- $\alpha$ ) levels are higher in the patients with Bell's palsy compared with the control group $[2,7]$. It was reported that there is increasing evidence about Bell's palsy occurring due to inflammation or autoimmune reaction in the facial nerve as a result of latent herpes virus reactivation [1, 2].
In terms of etiology, the TGd-MRI is usually necesseary, and the most common pathology is the contrast enhancement at the facial nerve distal intracanalicular portion and labyrinthine segment. A similar contrast enhancement can also be seen in the geniculate ganglion [2]. Kum et al. [4] conducted a retrospective study on patients with Bell's palsy and reported geniculate ganglion, distal intracranial segment, and labyrinthine segment enhancement. These contrast enhancements in the facial nerve were detected in only 3 out of 24 patients with Bell's paralysis in the present study. The contrast enhancement in the facial nerve was at the internal acoustic channel level in two of the patients and at the facial recess level in one of the patients.

NLR is frequently used as a new inflammation marker according to recent studies [3-5, 8]. NLR can be easily calculated with a CBC test. Bucak et al. [3] conducted a retrospective study in the patients with Bell's palsy and had showed that the neutrophil counts and the NLR value significantly increased in the patients with Bell's palsy compared to the control 
group. They also reported that the NLR was higher in the group with an unsatisfactory recovery than in the group with a satisfactory recovery, and therefore the NLR marker could be used as a marker to predict the disease prognosis [3]. Similarly, Kum et al. [4] found that the NLR values were significantly higher in those with Bell's palsy than in the control group. Moreover, the TGd-MRI results showed that the NLR was higher in patients with facial nerve involvement than in those without involvements, and that a positively significant correlation existed between the NLR value and facial paralysis stage. On the contrary, no correlation was detected with the MPV value. That study also suggested that these results provided evidence to support inflammation theory rather than microvascular theory in the etiopathogenesis of Bell's palsy [4]. Özler et al. [9] showed that the NLR value was high in Bell's palsy, and that a positive correlation existed between the NLR value and the level of paralysis. Moreover, they reported that high NLR value was a marker of poor prognosis [9]. Tekgul et al. [6] had showed that both B-cells (CD19) and T helper (CD4) values decrease in Bell's palsy. Nevertheless, they had reported that there was no prognostic significance of lymphocyte subset analysis in peripheral blood to predict the outcome of patients with unsatisfactory recovery [6]. In a retrospective study conducted by Atan et al. [10], NLR and PLR values were higher in the group of patients with Bell's palsy, but they found no correlation between these values and the level of facial paralysis [10]. Sahin et al. [11] had showed that neutrophil counts and NLR value increase with in Bell palsy patients but there were no statistical significant changes of PLR, MPV and PDW values. Additionally, they had not detect of association between the NLR value and grade of Bell's palsy (HB grade) [11]. A study of pediatric patients showed that the neutrophil count and NLR was higher in the Bell's palsy group, but no correlation was found between NLR and the grade of facial paralysis [12]. Wasano et al. reported that neutrophil count and the NLR value were higher in unrecovered Bell's palsy while the lymphocyte count reduced. They had also reported a correlation between the changes in blood test values and the prognosis of facial paralysis as a consequence of viral infection's impact on the bone marrow [5]. In another study, Wasano et al. [13] had defined to prognosis prediction score system for Bell's palsy. This scoring system includes the age ( $\geq 65$ years), gender (male) and NLR value $(\geq 2.9)$ of the patients. They had reported that the palsy prognosis prediction scores are useful for predicting prognosis of Bell's palsy before beginning treatment [13]. In this study, the NLR value was significantly higher in the patient group, which is consistent with the previous studies. Additionally, PDW and WBC were found to be higher in the patient group. These results might support the inflammation theory in patients with Bell's palsy. Unlike previous studies, no correlation was found between the NLR value and theprognosis. This result might be due to the small number of unrecovered patients in this study. MPV and PDW markers have been representing the platelet activation [14-16]. The MPV value shows the size of platelets while the PDWvalue is used to evaluate variations in platelet size. An increase in these markers suggests that bone marrow has released larger-volume platelets into blood circulation. Platelets with a large volume have been showed to be metabolically and enzymatically more active and more tendencies to aggregation compared with the small ones [15-17]. Özler et al. [18] had showed that the MPV and the PDW values in patients with Bell palsy were higher. Additionally, they had showed that there was positive correlation between PDW and MPV values with grade of facial paralysis. However, they had reported that there was no correlation between MPV and PDW values and prognosis of facial paralysis [18]. In contrast, Kum et al. [4] had reported no correlation with between the MPV value and grade of facial paralysis. In this study, the platelet count was significantly lower, the PDW value was higher, and the MPV value was higher (statistically not significant) in the recovered patients. When the recovered and unrecovered patients were compared according to the cut-off values in the ROC curve, no significant difference was found in the NLR and WBC values, but the possibility of not recovering was found to be significantly high in the patients with normal PDW values.The result might support the microcirculatory insufficiency theory in patients with Bell's palsy.

\section{Limitations}

The limitation of this study is the small number of patients because this is prospective and a single-center study. 


\section{CONCLUSION}

Based on these results, this study showed that bone marrow functions may be affected in patients with Bell's palsy; however, there is need for prospective studies that more patients are included to confirm this possibility.

\section{Conflict of interest}

The authors disclosed no conflict of interest during the preparation or publication of this manuscript.

\section{Financing}

The authors disclosed that they did not receive any grant during conduction or writing of this study.

\section{Acknowledgements}

We would like to thank Assoc. Prof. Dr. Serdal Kenan Köse for the statistical analysis of the work.

This study was presented as E-poster at the $38^{\text {th }}$ Turkish National Congress of OtorhinolaryngologyHead and Neck Surgery. 26-30 October 2016, Antalya, Turkey

\section{REFERENCES}

[1] Finsterer J. Management of peripheral facial nerve palsy. Eur Arch Otorhinolaryngol 2008; 265:743-52.

[2] Greco A, Gallo A, Fusconi M, Marinelli C, Macri GF, de Vincentiis M. Bell's palsy and autoimmunity. Autoimmun Rev 2012;12:323-8.

[3] Bucak A, Ulu S, Oruc S, Yucedag F, Tekin MS, Karakaya F, et al. Neutrophil-to-lymphocyte ratio as a novel-potential marker for predicting prognosis of Bell palsy. Laryngoscope 2014; 124:1678-81.

[4] Kum RO, Yurtsever Kum N, Ozcan M, Yilmaz YF, Gungor $\mathrm{V}$, Unal A, et al. Elevated neutrophil-to-lymphocyte ratio in Bell's palsy and its correlation with facial nerve enhancement on MRI. Otolaryngol Head Neck Surg 2015;152:130-5.

[5] Wasano K, Kawasaki T, Yamamoto S, Tomisato S, Shinden $\mathrm{S}$, Ishikawa T, et al. Pretreatment hematologic findings as novel predictive markers for facial palsy prognosis. Otolaryngol Head
Neck Surg 2016;155:581-7.

[6] Tekgul H, Polat M, Serdaroğlu G, Ikizoğlu T, Yalaz M, Kutukculer N, et al. Lymphocyte subsets in Bell's palsy: immune pathogenesis and outcome prediction. Pediatr Neurol 2004;31:258-60.

[7] Yilmaz M, Tarakcioglu M, Bayazit N, Bayazit YA, Namiduru M, Kanlikama M. Serum cytokine levels in Bell's palsy. J Neurol Sci 2002;15:69-72.

[8] Küçük E, Kocayiğit I, Günel C, Düzenli H. Neutrophil-tolymphocyte ratio in occlusive vascular diseases: the literature review of the past 10 years. World J Emerg Med 2016;7:165-72.

[9] Özler GS, Günak G. Neutrophil-lymphocyte ratio: a new predictive and prognostic factor in patients with Bell palsy. J Craniofac Surg 2014;25:944-5.

[10] Atan D, Ikinciogullari A, Koseoglu S, Ozcan KM, Cetin MA, Ensari S, et al. New predictive parameters of Bell's palsy: neutrophil to lymphocyte ratio and platelet to lymphocyte ratio. Balkan Med J 2015;32:167-70.

[11] Sahin C, Varım C. Neutrophil to lymphocyte ratio, platelet to lymphocyte ratio, mean platelet volume and red cell distribution width measures in Bells palsy. Open Access Maced J Med Sci 2017;15:14-8.

[12] Eryilmaz A, Basal Y, Tosun A, Kurt Omurlu I, Basak S. The neutrophil to lymphocyte ratios of our pediatric patients with Bell's palsy. Int J Pediatr Otorhinolaryngol 2015;79:2374-7.

[13] Wasano K, Ishikawa T, Kawasaki T, Yamamoto S, Tomisato $\mathrm{S}$, Shinden $\mathrm{S}$ et al. Novel pre-therapeutic scoring system using patient and haematological data to predict facial palsy prognosis. Clin Otolaryngol 2017;42:1224-8.

[14] Chen Y, Xiao Y, Lin Z, Xiao X, He C, Bihl JC et al. The role of circulating platelets microparticles and platelet parameters in acute ischemic stroke patients. J Stroke Cerebrovasc Dis 2015;24:2313-20.

[15] Martin JF, Trowbridge EA, Salmon G, Plumb J. The biological significance of platelet volume: its relationship to bleeding time, platelet thromboxane B2 production and megakaryocyte nuclear DNA concentration. Thromb Res 1983;32:443-60.

[16] Vagdatli E, Gounari E, Lazaridou E, Katsibourlia E, Tsikopoulou F, Labrianou I. Platelet distribution width: a simple, practical and specific marker of activation of coagulation. Hippokratia 2010;14:28-32.

[17] Sevuk U, Bahadir MV, Altindag R, Baysal E, Yaylak B, Ay $\mathrm{N}$, et al. Value of serial platelet indices measurements for the prediction of pulmonary embolism in patients with deep venous thrombosis. Ther Clin Risk Manag 2015;11:1243-9.

[18] Özler GS, Okuyucu S. Increased mean platelet volume in patients with Bell's palsy. Int Adv Otol 2014;10: 281-3. 